\title{
Family physician referral rates for lumbar spine computed tomography in Newfoundland and Labrador: a cross-sectional analysis using routinely collected data
}

\author{
Gabrielle S. Logan MSc, Bethan Copsey PhD, Holly Etchegary PhD, Patrick Parfrey MD, \\ Krista Mahoney $\mathrm{PhD}$, Amanda Hall PhD
}

\section{Abstract}

Background: Reducing computed tomography (CT) examinations of the lumbar spine is one of Choosing Wisely Canada's initial top 10 recommendations. This study's objective was to report the age- and-sex standardized rates of lumbar spine CT ordered by family physicians in 1 health region in Newfoundland and Labrador.

Methods: We conducted a retrospective study using local health data from Meditech, an electronic health record system, from 2013 to 2016 for the Eastern Health Region of Newfoundland and Labrador, the largest health region in the province. Records were included if the referral was for an adult aged 20 years or more, and CT was ordered by a family physician. Lumbar spine CT rates were contextualized with age- and sex-stratified estimates. Population estimates were provided by the Newfoundland and Labrador Centre for Health Information to calculate age- and sex-standardized rates per 100000 people. We calculated rate ratios to test for statistical significance in differences in rates between years.

Results: A total of 14370 records were examined. The age- and sex-standardized rates of lumbar spine CT per 100000 were 1225 in 2013, 1393 in 2014, 1556 in 2015 and 1395 in 2016. The rate ratio was 1.137 (95\% confidence interval [Cl] 1.084-1.194) for the comparison between 2014 and 2013, 1.117 (95\% Cl 1.067-1.169) between 2015 and 2014, and 0.896 (95\% Cl 0.857-0.938) between 2016 and 2015.

Interpretation: The age- and sex-standardized rates suggest that there was a steady rate of lumbar spine CT examinations being ordered by family physicians in Newfoundland and Labrador in 2013-2016. Although all rate ratios were statistically significant, the magnitude of the difference between years is likely not clinically relevant. These rates are important because they serve as a benchmark for future initiatives to reduce unnecessary referrals for lumbar spine CT.

hoosing Wisely Canada recommends reducing unnecessary lumbar spine imaging for low back pain, primarily to improve patient safety by avoiding unnecessary exposure to carcinogenic ionizing radiation and secondarily to reduce health care spending associated with overtesting. ${ }^{1,2}$ However, only a handful of studies have examined population-based use of computed tomography (CT) examinations of the lumbar spine, with most studies reporting the proportion of patients with low back pain who receive CT imaging compared to those who do not. ${ }^{3,4}$ Australia and the United States provide population-level data on lumbar spine CT use, which is helpful for comparisons of use internationally., ${ }^{5,6}$ These estimates range from 209 to 2464 CT examinations per 100000 people..$^{5,6}$ In Canada, this type of data is challenging to find. The only data we could identify were provided in a government-commissioned report on appropriate imaging that focused on lumbar spine CT rates in
2 Canadian provinces, Manitoba and Ontario; different estimates were found in the 2 provinces. ${ }^{7}$

The objective of the present study was to determine the yearly age- and sex-standardized rates of lumbar spine CT for adults ( $\geq 20 \mathrm{yr})$ ordered by family physicians in the Eastern Health Region of Newfoundland and Labrador, Canada. We focused on CT largely in response to a report by the Canadian Institute for Health Information indicating Newfoundland and Labrador's all-type CT rate to be twice the national average and the second highest among all the

Competing interests: None declared.

This article has been peer reviewed.

Correspondence to: Gabrielle Logan, glogan@mun.ca

CMAJ Open 2020. DOI:10.9778/cmajo.20190076 
provinces. ${ }^{8}$ Although we could hypothesize that the rate of lumbar spine CT is also higher in Newfoundland and Labrador than in other provinces, comparisons between provinces are beyond the scope of this study owing to lack of data required for this analysis.

\section{Methods}

\section{Setting and study period}

This study was conducted in the Eastern Health Region of Newfoundland and Labrador. It is the largest health region in the province, providing health care to about 300000 people, or $60 \%$ of the province's population. We chose 2013-2016 as the study period because these were years with data that were fully available, considered reliable and recent. Older, nondigitized data were unreliable because of a change in a data management system. No policy changes or educational campaigns to reduce imaging occurred during the study period.

\section{Data source}

The third-party data custodian, the Newfoundland and Labrador Centre for Health Information, identified the data set from the administrative codes for lumbar spine CT with and without contrast from Meditech, an electronic medical records database in the Eastern Health Region. Records from 2013 to 2016 were accessed and the following variables were collected: number of lumbar spine CT examinations with or without contrast, patient's age and sex, ordering physician specialty and imaging service date. Because only selected hospitals perform CT, we believe that this data set is comprehensive and accurate, encompassing all CT examinations performed with or without contrast in the Eastern Health Region during the study period.

\section{Inclusion criteria}

The data set contained all lumbar spine CT examinations conducted between Jan. 1, 2013, and Dec. 31, 2016. The inclusion criteria were adults ( $\geq 20 \mathrm{yr})$ who received a CT scan and referrals that were ordered by a family physician or a general practitioner (other specialties were excluded). Data that did not fit the inclusion criteria were removed. The pediatric population (< $18 \mathrm{yr})$ was removed because different diagnostic imaging guidelines apply to children than to adults. Patients aged 18-19 years were considered to be part of the pediatric group (group age range was 15-19) and would thus not appear in our data set. Therefore, we excluded the small number of patients $(n=98)$ aged 18-19 years in our adult data set since including them would underestimate the number of scans occurring in this age group, which would result in inaccurate rate estimations. We included only family physicians and general practitioners (hereafter referred to collectively as family physicians) because, in our local context, the first point of contact for a patient with low back pain typically is the patient's family physician. As such, we focused on this group of providers; this decision was made a priori. Finally, yearly totals of lumbar spine CT examinations were obtained.

\section{Outcomes}

We calculated crude rates per 100000 of lumbar spine CT referrals by dividing the total number of $\mathrm{CT}$ scans performed in the Eastern Health Region in each year of interest (numerator) by the total population of the Eastern Health Region in that same year of interest (denominator) and multiplying that proportion by 100000 . Population estimates were provided by the Newfoundland and Labrador Centre for Health Information. We used the 2015 population estimates to calculate the rate for 2016, as population estimates for 2016 were not available.

We calculated age- and sex-standardized rates of lumbar spine CT by categorizing all records of CT referrals into appropriate age groups and sex of the patient for each year of interest. Each age group contained 5 different age categories (e.g., 20-24 yr). We determined the CT rate for each year of interest for the applicable age or sex category by dividing the CT count for a given category by the population estimate for that same age or sex category and multiplying the proportion by 100000 .

We estimated CT counts for 2014, 2015 and 2016 using 2013 population age and sex estimates. For example, we calculated the count for 2014 by taking the 2014 rate for each age or sex category and dividing it by 100000 to get the proportion, then multiplying the proportion by the 2013 population estimate. Then we summed the estimated CT counts for each year of interest. We used the total estimate of CT counts for each year to calculate the age- and sex-adjusted rate by taking the CT count estimate for each year of interest, dividing it by the 2013 population estimate and multiplying it by 100000 .

\section{Statistical analysis}

To determine whether rates of CT referrals per 100000 people in a 1-year period increased over time, we calculated rate ratios and $95 \%$ confidence intervals (CIs). We compared each year's age- and sex-standardized lumbar spine CT rate to the previous year's rate to see whether there was a statistically significant change. Analyses were conducted with Microsoft Office 2010 Excel software.

\section{Ethics approval}

This study was exempt from ethical approval from the local health research ethics board as it was a secondary analysis of anonymized data and was part of a provincial qualityimprovement strategy. ${ }^{9}$

\section{Resullts}

A total of 18358 lumbar spine CT examinations were performed in the Eastern Health Region between 2013 and 2016. Of the 18358 records, 2831 were excluded because the health care provider was not a family physician (e.g., neurologist, orthopedic surgeon), 98 because of patient age and 1058 owing to insufficient information (missing physician specialty, or patient age or sex), resulting in 14370 included records. The average patient age was 54.1 years (standard deviation 
$14 \mathrm{yr}$ ), and 7795 (54.2\%) of the included CT referrals were for patients who identified as women. The numbers of included records stratified by age and sex are presented in Table 1.

The raw numbers of lumbar spine CT scans performed in the Eastern Health Region were 3118 in 2013, 3581 in 2014, 4042 in 2015 and 3629 in 2016. The crude rates of CT scans per 100000 were 1225 in 2013, 1399 in 2014, 1568 in 2015 and 1408 in 2016. The age- and sex-standardized rates per 100000 were similar in magnitude to the crude rates: 1225 in 2013, 1393 in 2014, 1556 in 2015 and 1395 in 2016.

The rate ratios comparing a given year to the previous year are presented in Table 2. The greatest increase in rates was between 2013 and 2014, and there was a decrease in rates between 2015 and 2016.

\section{Interpretation}

This study adds to the body of work in this area by presenting lumbar spine CT rates from Newfoundland and Labrador. The age- and sex-standardized lumbar spine CT rate per 100000 people ranged from 1225 to 1556 between 2013 and 2016. Although our rate ratio analysis identified that the observed differences in rates between years were statistically different, the magnitude of these differences was so small that they are likely clinically irrelevant, meaning that the difference in lumbar spine CT numbers for each year is most likely due to the large sample size and is not representative of actual changes in rates of CT ordering by family physicians. Thus, the lumbar spine CT rate in Newfoundland and Labrador remained steady from 2013 to 2016. Diagnostic imaging data from a larger time frame would allow for an accurate trend analysis.

Busse and colleagues ${ }^{7}$ searched the grey literature and found that, in Manitoba in 2010/11, the age- and sexstandardized rate of lumbar spine CT examinations ordered (all physicians included) was 967 (all axial CT scans) per 100000 people; in Ontario, the age- and sex-standardized rate was about 600 per 100000 people (all axial CT scans) (all physicians included). However, direct comparisons with our results are difficult, as we used the 2013 Newfoundland and Labrador Eastern Health population as the reference population in our analysis, and Busse and colleagues ${ }^{7}$ did not use the same reference population. It is also noteworthy that family physicians were the target provider in our study. This was not the case for Busse and colleagues. ${ }^{7}$ Those authors also collected data from earlier years than in our study; thus, changes may have occurred over time that would make comparison challenging.

For Australia, we found age-standardized rates of lumbar spine CT only; they varied from 209 to 2464 per 100000 people. ${ }^{6}$ In the US, different rates (age-, sex- and race-standardized) of spinal imaging (magnetic resonance imaging and CT) were reported from different hospital referral regions, ranging from 320 to 2370 per 100000 people. ${ }^{5}$

Caution needs to be taken when comparing rates of CT use for Newfoundland and Labrador to those for other countries. Although numerically our rates are within the range of
Table 1: Included patient referrals for lumbar spine computed tomography stratified by sex and age category

\begin{tabular}{|c|c|}
\hline Age category, yr & $\begin{array}{c}\text { No. of records } \\
n=14370\end{array}$ \\
\hline Female sex & 7795 \\
\hline $20-24$ & 132 \\
\hline $25-29$ & 249 \\
\hline 30-34 & 356 \\
\hline $35-39$ & 497 \\
\hline $40-44$ & 718 \\
\hline $45-49$ & 863 \\
\hline $50-54$ & 1051 \\
\hline $55-59$ & 1020 \\
\hline $60-64$ & 966 \\
\hline $65-69$ & 850 \\
\hline $70-74$ & 525 \\
\hline $75-79$ & 314 \\
\hline $80-84$ & 168 \\
\hline$\geq 85$ & 86 \\
\hline Male sex & 6575 \\
\hline $20-24$ & 119 \\
\hline $25-29$ & 209 \\
\hline $30-34$ & 338 \\
\hline $35-39$ & 414 \\
\hline $40-44$ & 577 \\
\hline $45-49$ & 742 \\
\hline $50-54$ & 869 \\
\hline $55-59$ & 918 \\
\hline $60-64$ & 880 \\
\hline $65-69$ & 636 \\
\hline $70-74$ & 457 \\
\hline $75-79$ & 237 \\
\hline $80-84$ & 128 \\
\hline$\geq 85$ & 51 \\
\hline
\end{tabular}

Table 2: Rate ratios comparing age- and sex-standardized computed tomography rate estimates in adjacent years

\begin{tabular}{|lc|}
\hline Year comparison & Rate ratio* $(95 \% \mathrm{Cl})$ \\
\hline 2014 to 2013 & $1.137(1.084-1.194)$ \\
\hline 2015 to 2014 & $1.117(1.067-1.169)$ \\
\hline 2016 to 2015 & $0.896(0.857-0.938)$ \\
\hline $\begin{array}{l}\text { Note: } \mathrm{Cl}=\text { confidence interval. } \\
{ }^{*} \text { Calculated by dividing the more recent year by the previous year. }\end{array}$ \\
\hline
\end{tabular}

those for other countries, differing population estimates for the reference populations and inclusion of magnetic resonance imaging in the US rates limits direct comparison. 
Although direct or indirect comparisons were beyond the scope of this paper, future research could look more closely at comparisons of lumbar spine CT use rates among provinces, especially given the high prevalence of low back pain, the potential harm to a patient from radiation and the questionable clinical utility for patients with uncomplicated low back pain who require only conservative care. ${ }^{2}$ Similarly, further research is needed to better understand how many CT scans are necessary for the management of a patient's condition. It is important to focus research on interventions targeting the health care system to improve the appropriateness of CT referrals, which would help ensure that patient safety is prioritized and health care funds are spent appropriately.

\section{Limitations}

It is important to note the limitations in our data set. First, the age- and sex-standardized rate for 2016 was based on population estimates from 2015. Given that the number of people in the Eastern Health Region may have changed from 2015 to 2016, the 2016 estimate may not be as robust as estimates with accurate data.

Second, the data used for analysis were routinely collected health data and were not collected with research purposes in mind. We cannot know whether there were misclassified or missing data, whether the quality and accuracy of the data were considered, or whether there were other unforeseen confounders (e.g., seasonal effects, local hospital policy change, rural patients travelling to the Eastern Health Region for care). ${ }^{10}$ There was no known policy change in the years included in the study; however, there may have been local changes that decreased the number of imaging examinations in 2016.

Third, repeat imaging of the same person may have occurred in any of the included years, which would have affected the imaging rate. Records that were missing patient age and specialist information were also excluded, which may have resulted in an underestimation of the true rate of CT. Specialist physicians were excluded from this analysis; the inclusion of these specialties would have increased the ageand sex-standardized rates of lumbar spine CT. We excluded from our analysis records with missing information, which may have led to underestimation of CT rates.

Finally, although it is possible that there may have been some overlap with thoracic spine CT, this is unlikely given the different codes that are used for such examinations. Repeated, cancelled and missed CT examinations may have occurred in the data set; these were not possible to distinguish from a completed examination and may have resulted in overestimation of the CT rates. This study did not assess the appropriateness of CT examinations or collect reasons for referrals. Future research will examine the appropriateness of referrals for lumbar spine CT.

\section{Conclusion}

There appears to have been a high rate of lumbar spine CT examinations ordered in the Eastern Health Region of Newfoundland and Labrador in 2013-2016, and this rate seems similar to or possibly even higher than those reported for other, larger Canadian provinces. These results provide valuable data benchmarks for any potential initiatives aimed at decreasing unnecessary referrals for lumbar spine CT.

\section{References}

1. Imaging tests for lower back pain: when you need them and when you don't. Toronto: Choosing Wisely Canada. Available: https://choosingwiselycanada. org/imaging-tests-low-back-pain/ (accessed 2019 Jan. 9)

2. Chou R, Deyo RA, Jarvik JG. Appropriate use of lumbar imaging for evaluation of low back pain. Radiol Clin North Am 2012;50:569-85.

3. Mafi JN, McCarthy EP, Davis RB, et al. Worsening trends in the management and treatment of back pain. FAMA Intern Med 2013;173:1573-81.

4. Eccles M, Steen N, Grimshaw J, et al. Effect of audit and feedback, and reminder messages on primary-care radiology referrals: a randomised trial. Lancet 2001;357:1406-9.

5. Lurie JD, Birkmeyer NJ, Weinstein JN. Rates of advanced spinal imaging and spine surgery. Spine 2003;28:616-20.

6. Computed tomography of the lumbar spine. In Australian atlas of healthcare variation series. Sydney (Australia): Australian Commission on Safety and Quality in Health Care; 2015. Available: www.safetyandquality.gov.au/wp-content/uploads/ 2015/11/SAQ201_03_Chapter2_v9_FILM_tagged_merged_2-3.pdf (accessed 2019 Jan. 9).

7. Busse J, Alexander PE, Abdul-Razzak A, et al. Appropriateness of spinal imaging use in Canada. Hamilton: Canadian Institutes of Health Research, ICES, Manitoba Health, McMaster University; 2013.

8. Medical imaging in Canada. 2012. Ottawa: Canadian Institute for Health Information; 2013. Available: www.cihi.ca/en/medical-imaging-in-canada-2012 (accessed 2019 Sept. 12).

9. Does your study require ethics review? St. John's: Newfoundland and Labrador Health Research Ethics Authority; modified 2018 Nov. 6. Available: www.hrea. ca/how-to-apply/ethics-review-required/ (accessed 2019 Sept. 12).

10. Benchimol EI, Smeeth L, Guttmann A, et al.; RECORD Working Committee. The REporting of studies Conducted using Observational Routinelycollected health Data (RECORD) statement. PLoS Med 2015;12:e1001885.

Affiliations: Faculty of Medicine (Logan, Etchegary, Parfrey, Mahoney, Hall), Memorial University of Newfoundland, St. John's, Nfld.; Centre for Statistics in Medicine (Copsey), Nuffield Department of Orthopaedics, Rheumatology and Musculoskeletal Sciences, University of Oxford, Oxford, UK; Primary Health Research Unit (Hall), Memorial University of Newfoundland, St. John's, Nfld.

Contributors: Amanda Hall, Gabrielle Logan and Krista Mahoney conceived and designed the study. Gabrielle Logan and Bethan Copsey analyzed the data. Gabrielle Logan drafted the manuscript. Gabrielle Logan and Amanda Hall interpreted the data. All of the authors revised the manuscript critically for important critical content, approved the final version to be published and agreed to be accountable for all aspects of the work.

Supplemental information: For reviewer comments and the original submission of this manuscript, please see www.cmajopen.ca/content/8/1/ E56/suppl/DC1. 